\title{
Effets toxiques des herbicides à base du glyphosate sur les poissons et autres animaux aquatiques : approche bibliographique
}

\author{
Prudencio AGBOHESSI* et Ibrahim IMOROU TOKO
}

\author{
Laboratoire de Recherche en Aquaculture et Ecotoxicologie Aquatique (LaRAEAq) \\ Faculté d'Agronomie (FA), Université de Parakou, BP : 123 Parakou, République du Bénin. \\ *Auteur correspondant ; E-mail : agbohessip@yahoo.fr; Tél. :0022996497616
}

Received: 08-01-2021

Accepted: 11-06-2021

Published: 31-12-2021

\section{RESUME}

Le glyphosate est un herbicide total foliaire systémique dont le principe actif est rarement utilisé seul mais le plus souvent mélangé à des formulants. Afin de faire la lumière sur ses effets toxicologiques sur les écosystèmes aquatiques, un travail de synthèse documentaire nous a permis d'établir sa toxicité sur les poissons, les invertébrés aquatiques et les amphibiens. Ainsi les CL50-96 h (Concentrations létales médianes sur 96 h) sur les poissons varient de $0,03 \mathrm{mg} / \mathrm{L}$ (Ictalurus punctatus) à $975 \mathrm{mg} / \mathrm{L}$ (Phalloceros caudimaculatus). Les effets chroniques recensés sur les poissons sont, entre autres, l'altération de la mémoire, la réduction de la mobilité, les dommages sur les paramètres hématologiques et plusieurs lésions histopathologiques sur les branchies, le tube digestif, le foie et le rein. Chez les invertébrés, les CL50 varient de 3-100 mg m.a/L (Daphnia magna) à $147 \mathrm{mg}$ $\mathrm{m} . \mathrm{a} / \mathrm{L}$ (Ceriodaphnia dubia) avec, entre autres, comme effets chroniques les perturbations de la reproduction et du développement, les perturbations des fonctions cardiaques et des paramètres du stress oxydatif. Les CL50 varient chez les amphibiens de $78 \mathrm{mg} \mathrm{m.a/L} \mathrm{(Crinia} \mathrm{insignifera)} \mathrm{à} \mathrm{138,9} \mathrm{mg} \mathrm{m.a/L} \mathrm{(Rana} \mathrm{clamitans).} \mathrm{Au} \mathrm{total,}$ les effets toxiques sont induits par de fortes concentrations du polluant et ainsi le glyphosate est considéré par la majorité des auteurs, peu toxique à la faune aquatique.

(C) 2021 International Formulae Group. All rights reserved.

Mots clés : Glyphosate, herbicide, toxicité, faune aquatique.

\section{Toxic effects of glyphosate-based herbicides on fish and other aquatic animals: bibliographic approach}

\begin{abstract}
Glyphosate is a total foliar systemic herbicide, whose active ingredient is rarely used alone but more often mixed with formulants. In order to shed light on its toxicological effects on aquatic ecosystems, documentary synthesis has enabled us to establish its toxicity on fish, aquatic invertebrates and amphibians. Thus, LC50-96 h (Median Lethal Concentrations over $96 \mathrm{~h}$ ) on fish vary from $0.03 \mathrm{mg} / \mathrm{L}$ (Ictalurus punctatus) to $975 \mathrm{mg} / \mathrm{L}$ (Phalloceros caudimaculatus). Recurrent chronic effects in fish include impaired memory, reduced mobility, damage to haematological parameters and several histopathological lesions on the gills, digestive tract, liver and kidney. In invertebrates, LC50s vary from 3-100 mg ai / L (Daphnia magna) to $147 \mathrm{mg}$ ai / L (Ceriodaphnia dubia) with, among other chronic effects, disturbances of reproduction and development, disturbances of cardiac functions and parameters of oxidative stress. LC50s vary in amphibians from $78 \mathrm{mg}$ a.i /
\end{abstract}


L (Crinia insignifera) to $138.9 \mathrm{mg}$ a.i / L (Rana clamitans). In conclusion, the toxic effects are induced by high concentrations of the pollutant, consequently, glyphosate is considered by the majority of authors to be of low toxicity to aquatic fauna.

(C) 2021 International Formulae Group. All rights reserved.

Keywords: Glyphosate, herbicide, toxicity, aquatic fauna.

\section{INTRODUCTION}

Plusieurs types de pesticides sont utilisés dans le but de lutter efficacement contre les bio-agresseurs des cultures et d'améliorer les rendements (Faure et al., 2000 ; Agbohessi et al., 2011; Agbohessi et al., 2012). Au nombre de ceux-ci nous avons les herbicides appelés parfois désherbants (Naili, 2014). Ce sont des matières actives ou des produits formulés ayant la propriété de tuer les végétaux (Faure et al., 2000 ; Naili, 2014). Les deux molécules à propriétés phytocides utilisées à l'échelle paysanne les plus répandues en Afrique en général et particulièrement en Afrique de l'Ouest sont le glyphosate et l'atrazine, mais le glyphosate reste le plus vendu et le plus utilisé sur le plan mondial (Adéchian et al., 2015). Sa consommation a atteint 825804 tonnes métriques en 2014 (Benbrook, 2016). Selon une étude publiée dans la revue environnementale Sciences Europe, plus de 850000 tonnes de glyphosate, appelé aussi «Tueur de mauvaises herbes », ont été consommés en 2015 dans le monde, appliqués sur 360 millions d'hectares de terres agricoles. La France l'un des grands consommateurs du glyphosate a appliqué plus de 9000 tonnes en 2016 et 8673 tonnes en 2017. Il est le plus utilisé en terme de quantité notamment dans les champs de coton pour lutter contre les plantes adventices (Rainaud, 2013 ; Adéchian et al., 2015). Dérivé de la glycine, le glyphosate est un herbicide total foliaire systémique, c'est-à-dire non sélectif, pénétrant par les feuilles, puis transporté de manière systémique jusqu'aux racines (Druart et al., 2011). Cette molécule est rarement utilisée seule mais le plus souvent mélangée à des formulants car seule, elle n'adhère pas aux feuilles et les pénètre difficilement (Le Mer et al., 2009). Au Bénin où ce désherbant est très fortement utilisé, pour la seule campagne cotonnière 2013-2014, plus de 1241644 T/L d'herbicides totaux et plus de $1359000 \mathrm{~T} / \mathrm{L}$ d'herbicides sélectifs, le tout à base du glyphosate, ont été commandés pour la seule culture du coton (MAEP, 2015). Ces quantités énormes d'herbicides provenant du circuit formel ne représentent que $6,1 \%$ de la quantité totale utilisée par les paysans, le reste $93,9 \%$ représentant la part d'herbicides non autorisés provenant du circuit informel dans la commune de Gogounou (Adéchian et al., 2015). Il est aujourd'hui incontestable que ces produits utilisés dans les champs font des écosystèmes aquatiques leurs réceptacles finaux rendant vulnérables les espèces qui y vivent (Katrijn et al., 2007 ; Agbohessi et al., 2015). Des études ont montré dans l'eau à Ontario (Canada), des teneurs en glyphosate allant à $41 \mu \mathrm{g} / \mathrm{L}$ en 2004 et 30,5 $\mu \mathrm{g} / \mathrm{L}$ en 2005 (Struger et al., 2008). Des publications ont également rapporté en Belgique, en Irlande et en Suède des concentrations en glyphosate dans l'eau superficielle allant respectivement à $139 ; 186$ et $370 \mu \mathrm{g} / \mathrm{L}$. En Afrique au Bénin, la recherche de résidus d'aminophosphates dans l'eau et les sédiments de la rivière Agbado à Savalou a rapporté la présence du glyphosate allant respectivement de 0,10 à 1,316 ppb et jusqu'à $8,62 \mathrm{ppb}$ (Gbaguidi et al., 2011). A Djidja toujours au Bénin, des teneurs en résidus de glyphosate dans les eaux ont varié de 0,105 à 0,193 g/L (Aïkpo et al., 2015). Le glyphosate a fait l'objet ces dernières années de beaucoup de polémiques quant à sa toxicité sur l'environnement et sur l'homme. La présente étude qui est une synthèse de la littérature disponible, visait à faire la lumière sur les effets toxicologiques de ce phytocide encore très fortement utilisé en Afrique de l'Ouest en général et au Bénin en particulier, sur les écosystèmes aquatiques. Il s'agit de faire une revue documentaire sur ses caractéristiques 
physico-chimiques et ses effets aigus, sublétaux et chroniques sur les poissons et les autres organismes animaux des biotopes aquatiques.

\section{STRUCTURE CHIMIQUE ET MODE D'ACTION DU GLYPHOSATE SUR LES PLANTES ADVENTICES}

Le glyphosate, ou N-phosphonométhyl glycine, est un herbicide à large spectre appartenant à la famille des aminophosphonates (ACTA, 2010 ; INERIS, 2011). C'est un analogue de la glycine, un acide aminé naturel sur lequel on a substitué un atome d'hydrogène par un groupement phosphonométhyle au niveau de la fonction amine primaire (R-NH2) (Figure 1). Le glyphosate est un herbicide de post-émergence, non sélectif, avec un large spectre d'activité sur les plantes annuelles ou pérennes. Il est absorbé par la plante au niveau des feuilles puis est rapidement véhiculé par la sève jusqu'à l'extrémité des racines et des rhizomes (Solomon et Thompson, 2003 ; Picqué, 2016). La cible exacte du glyphosate dans la plante a été décrite. Le glyphosate inhibe la 5énolpyruvylshikimate-3-phosphate synthase (EPSP synthase), une enzyme essentielle dans la biosynthèse des acides aminés aromatiques (Picqué, 2016). L'EPSP synthase catalyse la formation de l'EPSP à partir du phosphoénolpyruvate (PEP) et du shikimate-3phosphate (S3P). Il s'agit d'une étape essentielle de la production du chorismate, substance requise pour la biosynthèse d'acides aminés aromatiques essentiels, du tétrahydrofolate, de l'ubiquinone et de la vitamine $\mathrm{K}$. Ces derniers sont tous des produits très importants (Picqué, 2016). Cette voie ne se trouve que chez les plantes et les microorganismes photosynthétiques. Aucune autre famille de phytocides commerciaux n'agit de la même manière que le glyphosate, et les produits élaborés pour tenter de reproduire la spécificité du glyphosate ne sont pas aussi efficaces (Picqué, 2016). Il est en effet capable de contrôler la présence de n'importe quelle plante adventice et permet d'éviter la multiplication des traitements. Cet aspect pratique fait de lui un herbicide très apprécié, aussi bien par les professionnels (agriculteurs, employés des collectivités, paysagistes, etc) que par les particuliers.

Dans l'environnement, le principal photoproduit formé à partir du glyphosate est l'acide aminométhylphosphonique (Figure 2) noté AMPA (Durand, 2012). La photodégradation reste cependant une voie mineure de disparition du glyphosate. De même, sa dégradation chimique (hydrolyse ou oxydation) ne semble que peu ou pas impliquée (INERIS, 2011). Le glyphosate peut être biodégradé par des bactéries telles que Flavobacterium, Pseudomonas sp, etc, ou par des champignons comme Penicillium citrinium, Penicillium chrysogenium, etc (Durand, 2012). Le principal produit obtenu est l'AMPA (INERIS, 2011 ; Durand, 2012).

\section{PROPRIETES PHYSICO-CHIMIQUES ET METHODES D'ANALYSE DU GLYPHOSATE \\ Propriétés physico-chimiques du glyphosate}

Les principales caractéristiques physico-chimiques du glyphosate et celles d'autres herbicides pour comparaison sont résumées respectivement dans les Tableaux 1 et 2. Le glyphosate est un acide faible organique amphotère, qui peut se trouver sous différentes formes ionisées en fonction du $\mathrm{pH}$. Il est difficilement utilisable. Cependant, des sels dont le sel d'isopropylamine, servent de support à cet acide permettant de rendre la matière active parfaitement soluble dans l'eau (INERIS, 2011). Le glyphosate et son sel d'isopropylamine se présentent sous la forme d'une poudre cristalline blanche inodore (INRS, 2019). Le glyphosate est modérément soluble dans l'eau $\left(10\right.$ à $12 \mathrm{~g} / \mathrm{L}$ à $20^{\circ} \mathrm{C}$ à $\mathrm{pH}=$ 2) et insoluble dans la plupart des solvants organiques (RCQE, 2012 ; INERIS, 2017 ; INRS, 2019). Mais par comparaison à la dizaine d'herbicide comme l'atrazine, l'alachlor, l'acetachlor, etc, listée dans le Tableau 2, le glyphosate semble plus soluble dans l'eau. Le sel d'isopropylamine quand à lui, est plus soluble dans l'eau (1050 g/L à 25 $\left.{ }^{\circ} \mathrm{C}\right)$ que le glyphosate (INRS, 2019). La tension de vapeur du glyphosate est faible et plus basse que les autres phytocides listés dans le Tableau 
2 (RCQE, 2012 ; INRS, 2019). Sa Constante de Henry $\left(2,1.10^{-7} \mathrm{~Pa} \cdot \mathrm{m}^{-3} / \mathrm{mol}\right)$ indique sa faible volatilité en solution aqueuse (EC, 2002; INERIS, 2011). Le glyphosate se dissipe rapidement dans l'eau où sa demi-vie varie de quelques jours à plusieurs semaines (Kennedy, 2017). Dans les étangs, sa demi-vie varie de 1,5 à 3,5 jours. La vitesse de dissipation du glyphosate dans l'eau semble être fonction de la teneur en sédiments, de la chimie de l'eau et de la photodégradation. Les sédiments constituent le principal puits pour les résidus de glyphosate dans l'eau (Schuette, 1998). Le glyphosate a un coefficient de partage carbone organique-eau très élevé $(28000 \mathrm{~mL} / \mathrm{g})$, plus élevé que celui des autres herbicides (Tableau 2), ce qui explique sa forte tendance réversible à passer préférentiellement de l'eau aux sédiments (RCQE, 2012; INRS, 2019). En outre, la demi-vie de dissipation du glyphosate semble être corrélée à l'alcalinité de l'eau, les plus longs temps de demi-vie étant observés dans les eaux les plus alcalines (Goldsborough et Brown, 1993). Par ailleurs, il existe des résultats discordants concernant l'adsorption du glyphosate sur les matières en suspension et les sédiments benthiques dans les cours d'eau. Certaines études indiquent de plus fortes quantités résiduelles de la substance dans l'eau comparativement à d'autres études. Selon Bowmer et al. (1986), aux concentrations supérieures à $0,5 \mathrm{~g} \mathrm{~m} . \mathrm{a} / \mathrm{m}^{3}$, moins de $30 \% \mathrm{du}$ glyphosate présent dans la colonne d'eau se lie aux particules en suspension.

La bioaccumulation du glyphosate dans le poisson n'est pas considérée comme significative (EC, 2002). Selon le coefficient de partage octanol/eau très faible et sa faible liposolubilité, le glyphosate ne devrait pas se bioaccumuler dans les organismes aquatiques (Kennedy, 2017). Le facteur de bioconcentration de la formulation Roundup serait de 1,6 pour le crapet arlequin Lepomis macrochirus (Tooby, 1985). Pour la carpe Cyprinus carpio et le tilapia rouge Oreochromis mossambicus, des facteurs de bioconcentration variant de 10,0 à 42,3 et de 12,0 à 35,4 respectivement ont été calculés (Wang et al., 1994).Tout cela montre la faible bioconcentration du glyphosate chez les organismes aquatiques (INERIS, 2011; Kennedy, 2017).

D'un point de vue chimique, le glyphosate attaque le fer et l'acier galvanisé en libérant de l'hydrogène, et peut réagir vivement avec les bases fortes en une réaction exothermique. Il se décompose par combustion en dégageant des fumées toxiques qui contiennent notamment des oxydes d'azote, des oxydes de phosphore et des oxydes de carbone (Kennedy, 2017 ; INRS, 2019). Chez la plupart des végétaux, le glyphosate n'est que faiblement dégradé après absorption. Cette stabilité métabolique permet à la molécule de disposer de tout le temps nécessaire pour atteindre l'extrémité des racines et exercer son effet (INRS, 2019).

\section{Propriétés physico-chimiques de l'AMPA}

En conditions tant aérobies qu'anaérobies, l'acide aminométhylphosphonique (AMPA) est le principal métabolite issu de la dégradation du glyphosate dans l'eau (INRS, 2019). Les autres métabolites représentent moins de $1 \%$ de la quantité initiale totale de glyphosate. Le Tableau 3 résume les caractéristiques physicochimiques de l'AMPA. La biodégradabilité de l'AMPA dépend du système eau / sédiment. Il reste entre 1 et $4 \%$ d'AMPA dans l'eau, après 100 jours (EC, 2002 ; INERIS, 2013). Il reste entre 20 et $32 \%$ d'AMPA dans les sédiments après 100 jours (EC, 2002). L'AMPA est biodégradé plus lentement que le glyphosate. D'après la valeur de Koc la substance est adsorbable (EC, 2002; INERIS, 2013). La valeur de constance de Henry suggère que l'AMPA n'a pas tendance à se volatiliser en solution aqueuse (INERIS, 2013). La bioconcentration de ce produit dans les organismes aquatiques est faible (US-EPA, 2008).

\section{Méthodes d'analyse du glyphosate}

Le glyphosate est fortement polaire, soluble dans l'eau et insoluble dans les solvants organiques. Par conséquent, plusieurs approches ont été élaborées pour l'analyser dans différentes matrices, telles l'eau, les sédiments, le sol, la végétation et les tissus 
animaux (Guo et al., 2005). Plusieurs méthodes, comme la chromatographie en phase gazeuse (CPG), la chromatographie liquide à haute performance (CLHP), la chromatographie ionique (CI), le test immunoenzymatique ELISA et l'électrophorèse capillaire (EC), permettent de déceler le glyphosate dans différents milieux (Stalikas et Konidari, 2001). L'analyse du glyphosate par CPG exige une étape supplémentaire pour rendre la substance moins polaire et assez volatile pour être injectée dans la colonne du CPG (Stalikas et Konidari, 2001). Puisque la technique de CPG exige beaucoup de temps, d'autres approches ont été proposées. Des méthodes couplant la chromatographie en phase gazeuse et la spectrométrie de masse (CPG-SM) ont été utilisées avec succès pour analyser la substance dans différents milieux (Stalikas et Konidari, 2001). Des limites de détection de 0,01 à $0,2 \mu \mathrm{g}$ $\mathrm{m} . \mathrm{a} / \mathrm{L}$ dans l'eau, de 6 à $50 \mu \mathrm{g} \mathrm{m} . \mathrm{a} / \mathrm{kg}$ dans le sol, de $50 \mu \mathrm{g} \mathrm{m} . \mathrm{a} / \mathrm{kg}$ dans des plantes cultivées et de 10 à $100 \mu \mathrm{g} \mathrm{m} . \mathrm{a} / \mathrm{kg}$ dans divers produits d'origine animale ont été relevées (Borjesson et Torstensson, 2000; Royer et al., 2000). Malgré la grande variété des techniques disponibles pour l'analyse du glyphosate, les méthodes chromatographiques demeurent les plus populaires (Stalikas et Konidari, 2001).

\section{EFFETS TOXIQUES DES HERBICIDES}

\section{A BASE DU GLYPHOSATE SUR LES} POISSONS

\section{Toxicités aigüe et sub-létale}

La toxicité d'un polluant sur un poisson dépend de l'espèce, son stade de développement, son état physiologique, son sexe, des caractéristiques physico-chimiques de l'eau dans laquelle vit le poisson. De façon globale, les résultats des essais de toxicité de courte durée sur une grande variété d'espèces indiquent que le glyphosate est relativement peu toxique (CCME, 2012). La toxicité du glyphosate peut être causée par les tensioactifs de la formulation, qui ont une toxicité similaire pour les organismes non-cibles (Arraj, 2015). Le Tableau 4 résume les CL50 après $96 \mathrm{~h}$ (concentrations létales médianes obtenues après $96 \mathrm{~h}$ d'exposition) du glyphosate sur différentes espèces de poisson. De la lecture de ce tableau, il ressort que les CL50 obtenues quelles que soient les conditions de l'essai, varient de $0,03 \mathrm{mg} / \mathrm{L}$ (Ictalurus. punctatus) à $975 \mathrm{mg} / \mathrm{L}$ (Phalloceros caudimaculatus). Les espèces plus sensibles au glyphosate sont, entre autres, I. punctatus $(0,03 \mathrm{mg} / \mathrm{L})$, Oncorhynchus keta $(0,04 \mathrm{mg} / \mathrm{L})$, Pimephales promelas $(0,0566 \mathrm{mg} / \mathrm{L})$ et Oncorhynchus gorbuscha (0,0567 mg/L) (RCQE, 2012). La toxicité du glyphosate est liée à la sensibilité des pesticides organophosphorés à l'inhibition de l'acétylcholinestérase. La toxicité des pesticides organophosphorés est due à l'inhibition du système nerveux central par la transformation physiologique du groupe phosphothioate $(\mathrm{P}=3)$ analogue du groupe $(\mathrm{P}=0)$ au niveau du pesticide (Al-Kawaz, 2019). Le groupe $(\mathrm{P}=0)$ inhibe l'action de l'acétylcholinestérase qui joue un rôle important dans la transmission de l'influx nerveux provoquant une obstruction de la neuropathie et la perte des poissons (Ani et al., 2017 ; Al-Kawaz et al., 2019). Les espèces les moins sensibles sont, entre autres, Tilapia zillii (211,80 mg/L), Cyprinodon variegatus $(240$ $\mathrm{mg} / \mathrm{L}$ ), Ovontesthes bonariensis (> $400 \mathrm{mg} / \mathrm{L}$ ), Cyprinus carpio $(620 \mathrm{mg} / \mathrm{L})$ et $P$. caudimaculatus $(975 \mathrm{mg} / \mathrm{L})$. Les valeurs des CL50 obtenues pour Clarias gariepinus varient de 0,05 à 43,65 mg/L selon le stade de développement du poisson. Nous avons trouvé nous-mêmes, suite à une exposition en 96 h d'alevins de poisson-chat africain au glyphosate en condition semi-statique avec renouvellement des solutions d'essai à $50 \%$ toutes les $48 \mathrm{~h}$ une CL50 de 13830 ppm (Données non encore publiées). Chez les tilapias du Nil Oreochromis niloticus les CL50 varient de 1,05 à $16,8 \mathrm{mg} / \mathrm{L}$. Des concentrations de 3 à $20 \mathrm{mg} / \mathrm{L}$ de glyphosate suite à une exposition sur $96 \mathrm{~h}$ des bogas Leporinus obtusidens provoquent une baisse significative de l'activité de l'acétylcholinestérase dans le cerveau et de la lactate dans le foie, une augmentation significative du glycogène et du glucose dans le foie ainsi que du taux d'ammoniac à la fois dans les muscles et dans le foie (Glusczak et al., 2006). Une étude exposant des carpes au 
Roundup en concentrations létales, a révélé que le glyphosate causerait une apparition de structures tels que des myélines dans les hépatocytes, un gonflement des mitochondries et une disparition de la membrane externe des mitochondries (Szarek et al., 2000).

Les réponses sub-létales des individus exposés au glyphosate varient d'une espèce à une autre. De façon globale, on note dès le début de l'exposition et successivement: des mouvements erratiques, une hyperactivité des individus, des mouvements operculaires rapides, des mouvements de la bouche à la surface de l'eau, le surfaçage, l'hypoactivité, l'épuisement et la mort de l'individu (Ibrahim et Khaled, 2017). Plusieurs autres auteurs ont noté chez certaines espèces d'autres réactions comme des stress respiratoires et des nages en flèche chez $C$. gariepinus (Okwara et al., 2016), une décoloration de la peau et des branchies et une hémorragie chez Lates calcarifer, une perte de l'équilibre et une courbure du corps dans la région caudale chez Carassus auratus (Al- Kawaz, 2019). Ces signes neurologiques montrent la neurotoxicité du glyphosate qui se manifeste par l'inhibition de l'activité de l'acétylcholinestérase sur le système nerveux central des poissons. L'effet sur la respiration observé chez certains poissons est l'inhibition par le glyphosate des enzymes impliquées dans la régulation des processus respiratoires. Les niveaux d'efficacité de la succinate déshydrogénase et de la malate déshydrogénase dans le foie et le cerveau sont réduits et provoquent une hypoxie dans les tissus (Al-Kawaz, 2019).

\section{Toxicité chronique}

Concernant l'effet chronique sur les poissons, les résultats sont controversés. La toxicité du Roundup testée sur plusieurs espèces de poisson, est considérée comme très faible par certains (Mitchell et al., 1987) alors que d'autres mettent en évidence des effets toxiques du glyphosate et de ses différentes formulations (Smith, 2001; Jiraungkoorskul et al., 2003; Tsui et Chu, 2003). Par exemple, une étude réalisée sur les carpes a mis en évidence que le Roundup provoque l'apparition de structures pathologiques au niveau des hépatocytes et notamment au niveau des mitochondries, et ceci à des concentrations 20 à 40 fois plus faibles que celles utilisées en pratique (Szarek et al., 2000). L'exposition à une dose de $0,5 \mathrm{mg} / \mathrm{L}$ et à 0,065 et $0,5 \mathrm{mg} / \mathrm{L}$ du Roundup réduirait la mobilité et les comportements agressifs chez les adultes des poissons zèbre Danio rerio (Bridi et al., 2017). La dose de $0,5 \mathrm{mg} / \mathrm{L}$ de Roundup entraînerait une altération significative de la mémoire chez cette espèce (Bridi et al., 2017). Plusieurs auteurs (Szarek et al., 2000 ; Glusczak et al., 2007 ; do Carmo Langiano et al., 2008) ont affirmé que de fortes concentrations de glyphosate induiraient des altérations histologiques dans le foie des poissons perturbant le fonctionnement normal de l'organe. Messaad et Zailaie (2017) ont observé chez Aphanus dispar exposé pendant 2 semaines à une dose de $28,81 \mathrm{mg} / \mathrm{L}$ de glyphosate, des œdèmes des hyperplasies associées à la fusion des lamelles secondaires, l'amincissement et le raccourcissement des lamelles secondaires au niveau des branchies, des nécroses, l'hypertrophie des hépatocytes, une vacuolisation du cytoplasme avec un aspect mousseux et une présence de noyaux pycnotiques au niveau du foie. Chez le tilapia du Nil soumis à une concentration de 1,05 $\mathrm{mg} / \mathrm{L}$ pendant 4 jours, Ayoola (2008) a relevé au niveau des branchies une prolifération des filaments dans les cellules, une fusion des lamelles, une hyperplasie des lamelles. Au niveau du foie, il a été noté une vacuolisation des hépatocytes et des nécroses, tandis qu'au niveau du rein une lésion constituée de gouttelettes hyalines dans le tube des cellules épithéliales, des picnoses et sur le cerveau, une dégénérescence neuronale généralisée et une spongiose. Des augmentations de l'activité de plusieurs enzymes dans le rein et le foie des carpes, ainsi que de l'hyperplasie épithéliale ont été observées. Le taux plasmique du glucose indiquant une réponse typique au stress ainsi que l'activité catalytique hépatique, suggérant l'activation des défenses antioxydantes, seraient élevés chez une espèce de poissons néo-tropicaux Prochilodus lineatus après une exposition à $10 \mathrm{mg} / \mathrm{L}$ de glyphosate (do Carmo Langiano et al., 2008). 
Une étude de 28 jours d'exposition à une dose de $0,5 \mathrm{mg} / \mathrm{L}$ au glyphosate a révélé l'altération des branchies, du foie et de l'activité sexuelle chez Jenynsia multidentata (Huet et al., 2012). Certaines lésions histologiques du tube digestif comme l'hyperplasie de la muqueuse gastrique, l'hyperplasie nécrotique, les modifications dégénératives et nécrotiques sévères de la muqueuse intestinale et de la sous-muqueuse ont été révélées chez $C$. gariepinus soumis à des concentrations de 18 à $72 \mathrm{mg} / \mathrm{L}$ du glyphosate pendant 2 mois (Erhunmwunse et al., 2014). Le glyphosate altère les paramètres hématologiques comme l'hémoglobine, le taux d'hématies et de leucocytes chez les heteroclarias (Adekunle et al., 2017), chez C. carpio (Ramesh et al., 2009), chez C. gariepinus (Okwara et al., 2016). D'autres paramètres hématologiques tels que le glucose, le cholesterol, les triglycérides, les protéines, l'albumine et les globulines sont aussi altérés chez Catla catla exposé pendant 4 jours à une concentration de $0,42 \mathrm{ppm}$ de glyphosate (Jerald et al., 2016). Dernièrement, une étude a montré que des concentrations de glyphosate $(0,2$ et $0,4 \mathrm{mg} / \mathrm{L})$ affecteraient les paramètres métaboliques et la production de TBARS dans le foie de Rhamdia quelen juvéniles (Glusczak et al., 2007). ElShebly et El-Kady (2008) ont indiqué une baisse de l'hormone de croissance et du taux de protéines musculaires chez $O$. niloticus exposé à des concentrations de 1 à $5 \mathrm{mg} / \mathrm{L}$ de glyphosate pendant 4 jours. Des expositions à long terme du glyphosate à des concentrations de 5 et $15 \mathrm{ppm}$ ont montré des effets histopathologiques et biochimiques chez $O$. niloticus (Jiraungkoorskul et al., 2003). Récemment, Çavas et Konen (2007) ont relevé que des concentrations de 5,10 et $15 \mathrm{ppm}$ du glyphosate causeraient des dommages dans les érythrocytes des poissons rouges $C$. auratus. Cependant, le glyphosate n'induit pas d'effet oestrogénique chez l'épinoche Gasterosteus aculeatus à des concentrations environnementales réalistes (Le Mer, 2009). Le potentiel mutagène du glyphosate a été évalué dans de très nombreux tests in vitro et in vivo, qui n'ont pas montré d'effets génotoxiques (INRS, 2019). Les différentes études de toxicité chronique réalisées n'ont pas mis en évidence de potentiel cancérogène pour le glyphosate (INRS, 2019). Aucun effet sur les paramètres de reproduction n'a été noté, et les études conduites n'ont pas mis en évidence d'effets tératogènes du glyphosate à des doses non toxiques pour la mère (INRS, 2019).

\section{EFFETS TOXIQUES DES HERBICIDES}

\section{A BASE DU GLYPHOSATE SUR LES} AUTRES ANIMAUX AQUATIQUES

Le Tableau 5 présente les CL50 du glyphosate chez quelques invertébrés aquatiques et chez quelques amphibiens. Les invertébrés ne sont pas très sensibles à la toxicité du glyphosate suite à une exposition de courte durée. Daphnia magna est l'espèce qui a été la plus étudiée et qui présente des valeurs variant de 3 à plus de $1000 \mathrm{mg}$ m.a/L pour la CL50/CE50 après $48 \mathrm{~h}$ respectivement associée à la mortalité ou l'immobilisation (US EPA, 2007a, 2007b et 2007c). Des valeurs de la CL50/CE50 après $48 \mathrm{~h}$ variant de 13 à $55 \mathrm{mg}$ m.a/L ont été publiées pour le moucheron Chironomus plumosus (US EPA, 2007b et 2007c). Une étude a révélé une valeur de CL50 après $48 \mathrm{~h}$ de 17,431 mg/L pour Artemia franciscana. Daphnia pulex a démontré une sensibilité au glyphosate similaire à celle de Daphnia magna, soit des valeurs de la CL50/CE50 après $48 \mathrm{~h}$ variant de 7,9 à 242 mg/L (US EPA, 2007b). Certaines espèces d'invertébrés sont particulièrement insensibles, entre autres Gammarus pseudolimnaeus qui présente des valeurs de la CL50 après $48 \mathrm{~h}$ variant de 42 et $62 \mathrm{mg} \mathrm{m} . \mathrm{a} / \mathrm{L}$ (US EPA, 2007b) et Mysidopsis bahia (un crustacé mysidacé) qui présente une CL50 après $96 \mathrm{~h}$ de $40 \mathrm{mg} \mathrm{m} . \mathrm{a} / \mathrm{L}$ (US EPA, 2007a). La base de données de l'EPA sur les pesticides à usage restreint (US EPA, 2007c) fait état de valeurs de 2,1 et $96 \mathrm{mg}$ m.a/L pour la CMEO après 21 jours et de 1,2 et $50 \mathrm{mg}$ m.a./L pour la CSEO dans le cas de Daphnia magna. James Elphick (Summit Environmental Consultants Ltd., 2007) a indiqué pour Hyalella azteca une CE10 après 14 jours de $53,9 \mathrm{mg} \mathrm{m} . \mathrm{a} / \mathrm{L}$ pour la survie et une CI10 pour le poids sec de $20,5 \mathrm{mg} \mathrm{m}$.a/L. Cette valeur se rapproche de la plage des valeurs rapportées pour Ceriodaphnia dubia et 
témoigne du peu de sensibilité de ces espèces au glyphosate.

Une toxicité suite à une exposition de courte durée au glyphosate à l'égard des amphibiens d'eau douce a été signalée pour quelques espèces. Des valeurs de la CL50 après $96 \mathrm{~h}$ de $78 \mathrm{mg} \mathrm{m} . \mathrm{a} / \mathrm{L}$ pour la grenouille australienne Crinia insignifera (US EPA, 2007a et 2007b) et de 11,6 et 110,8 mg m.a/L pour l'espèce Litoria moorei (US EPA, 2007a) ont été publiées. Les CL50 après $24 \mathrm{~h}$ et après $96 \mathrm{~h}$ pour la grenouille verte Rana clamitans dépasseraient 38,9 mg a.i/L (Howe et al., 2004). Plusieurs études récentes ont indiqué que les amphibiens sont l'un des groupes de vertébrés les plus sensibles aux effets toxiques du glyphosate. La CL50 pour de nombreux amphibiens se situe entre 1 et $10 \mathrm{mg} / \mathrm{L}$ (Govindarajulu, 2008).

Comme effets chroniques chez les invertébrés aquatiques, il fut démontré que le glyphosate affecte la reproduction et le développement des escargots Pseudosuccinea columella à des concentrations sous-létales (Tate et al., 1997) ainsi que le cycle de régulation des oursins Sphaerechinus granularis (Marc et al., 2002). Également, la fonction cardiaque et les paramètres du stress oxydatif des têtards de grenouille Lithobates catesbeianna seraient perturbés après une exposition de $48 \mathrm{~h}$ à $1 \mathrm{ppm}$ de glyphosate (Costa et al., 2008). Une exposition de cette espèce au glyphosate $(18 \mathrm{mg} / \mathrm{L})$ réduirait le taux de glycogène et de triglycérides dans les branchies, le foie et les muscles et une augmentation de la peroxydation lipidique et du cholestérol au niveau des branchies et des muscles, une baisse du taux de protéines a été également notée (Dornelles et Oliveira, 2015). Il fut démontré que le glyphosate $(1 \mathrm{mg} / \mathrm{L})$ provoquerait une croissance plus rapide à la troisième génération d'embryons d'escargots (Tate et al., 1997). Il a été également noté que des concentrations de 0,1 et $10 \mathrm{mg} / \mathrm{L}$ de glyphosate affecteraient la reproduction des escargots Pseudosuccinea columella en induisant des anormalités et des polyembryonies ainsi que l'éclosion serait inhibée à $10 \mathrm{mg} / \mathrm{L}$ (Tate et al., 1997). Une étude sur ce même gastropode, considérant le succès d'éclosion après 12 jours d'exposition, a indiqué une CMEO de $10 \mathrm{mg} \mathrm{m} . \mathrm{a} / \mathrm{L}$ et une CSEO de $1 \mathrm{mg} \mathrm{m.a/L} \mathrm{(Tate} \mathrm{et} \mathrm{al.,} \mathrm{1997).}$ Paunescu et Ponepal (2011) ont observé chez Pelophylax ridibundus exposé à une dose de $0,138 \cdot 10^{-3} \mathrm{~mL} / \mathrm{g}$ de poids corporel, une diminution du nombre d'érythrocytes et de glycémie, une augmentation du cholestérol plasmique et des triglycérides, une augmentation du nombre de leucocytes et de la valeur de l'indice hepato-somatique à une température de $22-24{ }^{\circ} \mathrm{C}$. Une étude d'essai chronique réalisée sur le développement embryonnaire et larvaire de la coquille SaintJacques Pecten maximus, a révélé que le glyphosate provoque une diminution du taux d'éclosion à de faibles doses, environ $25 \%$ de baisse à $0,5 \mu \mathrm{g} / \mathrm{L}$ et $45 \%$ à $1 \mu \mathrm{g} / \mathrm{L}$ (LarvorCario et al., 2000).

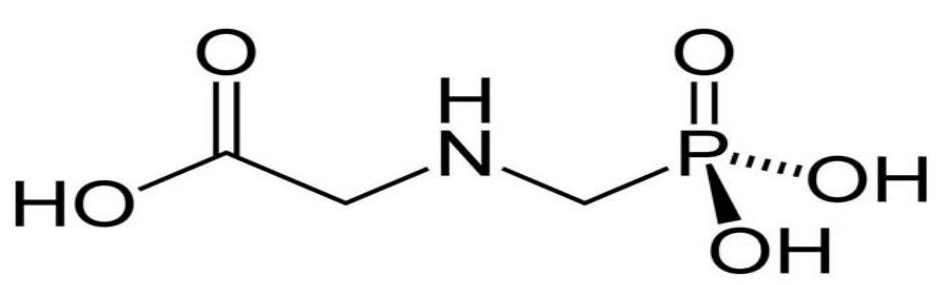

Figure 1 : Structure moléculaire du glyphosate. 


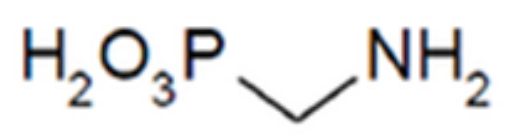

Figure 2 : Structure moléculaire de l'AMPA.

Tableau 1 : Synthèse des caractéristiques physico-chimiques du glyphosate.

\begin{tabular}{|c|c|c|}
\hline & Valeurs & Sources \\
\hline Numéro CAS & $1071-83-6$ & \multirow{3}{*}{$\begin{array}{c}\text { Naili, } 2014 \\
\text { Picqué, } 2016\end{array}$} \\
\hline Formule moléculaire & $\mathrm{C}_{3} \mathrm{H}_{8} \mathrm{NO}_{5} \mathrm{P}$ & \\
\hline Poids moléculaire $(\mathrm{g} / \mathrm{mol})$ & 169,1 & \\
\hline Densité (g/mol) & 1,74 & \multirow{11}{*}{$\begin{array}{c}\text { EC, } 2002 \\
\text { RCQE, } 2012 \\
\text { Naili, } 2014 \\
\text { Picqué, } 2016 \\
\text { Kennedy, } 2017\end{array}$} \\
\hline Pression de vapeur $(\mathrm{Pa})$ & $1,31 \cdot 10^{-5}$ à $25^{\circ} \mathrm{C}$ & \\
\hline Hydrosolubilité (mg/L) & 10500 à $20^{\circ} \mathrm{C}$ et $\mathrm{pH} 2$ & \\
\hline Coefficient de partage & $-4,59--1,7$ & \\
\hline Octanol/eau (Log/kow) & & \\
\hline $\begin{array}{l}\text { Solubilité dans les solvants } \\
\text { organiques }\end{array}$ & Insoluble dans la plupart des solvants & \\
\hline $\begin{array}{l}\text { Coefficient d'adsorption Koc } \\
(\mathrm{L} / \mathrm{kg})\end{array}$ & $884-60000$ & \\
\hline Constante de dissociation (pka) & $\begin{array}{l}\text { pKa1 : 2,34 à } 20^{\circ} \mathrm{C} \text { (Acide phosphate) } \\
\text { pKa2 : 5,73 à } 20^{\circ} \mathrm{C} \text { (Amine secondaire) } \\
\text { pKa3 : } 10,2 \text { à } 20^{\circ} \mathrm{C} \text { (Acide carboxylique) }\end{array}$ & \\
\hline $\begin{array}{l}\text { Constante de Henry } \\
\text { (Pa.m3/mol) }\end{array}$ & $2,1 \cdot 10^{-7}$ & \\
\hline Stabilité & $\begin{array}{l}\text { DT50 eau }=2 \text { à } 91 \text { jours } \\
\text { DT50 sol }=3 \text { à } 174 \text { jours }\end{array}$ & \\
\hline Point de fusion $\left({ }^{\circ} \mathrm{C}\right)$ & 200 & \\
\hline
\end{tabular}

Tableau 2 : Caractéristiques physico-chimiques d'autres herbicides (Obiora, 2008).

\begin{tabular}{ccccc}
\hline Herbicides & $\begin{array}{c}\text { Hydrosolubilité } \\
(\mathbf{m g} / \mathbf{L}) \mathbf{a} \mathbf{~ 2 5}^{\circ} \mathbf{C}\end{array}$ & $\begin{array}{c}\text { Coefficient de } \\
\text { partage octanol/eau } \\
(\text { Kow })\end{array}$ & $\begin{array}{c}\text { Coefficient } \\
\text { d'adsorption } \\
(\text { Kow })(\mathbf{L} / \mathbf{k g})\end{array}$ & $\begin{array}{c}\text { Pression de } \\
\text { vapeur }(\mathbf{m m H g}) \\
\mathbf{a ̀ ~ 2 5}^{\mathbf{}} \mathbf{C}\end{array}$ \\
\hline Acetachlor & 223 & 3,03 & 176,4 & $2,8.10^{-5}$ \\
\hline Alachlor & $148-242$ & $2,64-3,52$ & $43-209$ & $2,1.10^{-5}$ \\
\hline Atrazine & $20-35$ & $2,34-2,80$ & $38-288$ & $2,9.10^{-7}$ \\
\hline Cyanazine & $155-170$ & $2,02-2,24$ & $40-235$ & $1,4.10^{-7}$ \\
\hline Glyphosate & $10-157000$ & $-4,6--1,6$ & $9-60000$ & $4,3.10^{-10}$ \\
\hline Metolachlor & $488-550$ & $2,6-3,28$ & $22-307$ & $3,1.10^{-5}$ \\
\hline Metribuzyne & $1000-1220$ & $1,6-1,7$ & $3-95$ & $4,4.10^{-7}$ \\
\hline Prometon & $620-750$ & $2,69-2,99$ & $32-300$ & $2,3.10^{-6}$ \\
\hline Propazine & $3-8,6$ & $2,93-3,01$ & $100-603$ & $1,31.10^{-7}$ \\
\hline Simazine & $3,5-8,4$ & $2,10-2,18$ & $103-230$ & $2,21.10^{-8}$ \\
\hline
\end{tabular}


Tableau 3: Caractéristiques physico-chimiques de l'AMPA.

\begin{tabular}{|c|c|c|}
\hline & Valeurs & Sources \\
\hline Numéro CAS & $1066-51-9$ & INERIS, 2011 \\
\hline Formule moléculaire & $\mathrm{CH}_{6} \mathrm{NO}_{3} \mathrm{P}$ & \\
\hline Poids moléculaire (g/mol) & 111,04 & US-EPA, 2008 \\
\hline Hydro-solubilité (mg/L) & 1. $10^{-6}$ à $25^{\circ} \mathrm{C}$ & \\
\hline Pression de vapeur $(\mathbf{P a})$ & 0,0168 & \\
\hline Constante de Henry (Pa.m3/mol) & $1,27.10^{-10}$ à $25^{\circ} \mathrm{C}$ & \\
\hline $\begin{array}{l}\text { Coefficient de partage Octanol/eau (Log } \\
\text { Kow) }\end{array}$ & $-3,36--2,17$ & Traas et Smit, 2003 \\
\hline $\begin{array}{l}\text { Coefficient de partage carbone } \\
\text { organique/eau (L/kg) }\end{array}$ & $1160-248000$ & EC, 2002 \\
\hline Constante de dissociation pka & $\begin{array}{c}\text { pka1 }: 0,9 \\
\text { pka2 }: 5,6 \\
\text { pka3 }: 10,2\end{array}$ & Smit et al., 2001 \\
\hline
\end{tabular}

Tableau 4 : CL50 du glyphosate sur quelques espèces de poisson.

\begin{tabular}{|c|c|c|}
\hline Espèces & CL50-96 h (mg m.a/L) & Sources \\
\hline Ictalurus punctatus & 30,015 & \multirow{5}{*}{ RCQE, 2012} \\
\hline Lepomis macrochirus & 67,368 & \\
\hline Oncorhynchus gorbuscha & 56,711 & \\
\hline Oncorhychus keta & 42,372 & \\
\hline Oncorhynchus kisutch & 73,206 & \\
\hline Oncorhynchus mykiss & $68,48-140$ & Folman et al., 1979 \\
\hline Oncorhynchus tshawytsha & 66,747 & \multirow{2}{*}{ RCQE, 2012} \\
\hline Pimephales promelas & 56,632 & \\
\hline Cyprinodon variegatus & 240 & US EPA, 2005 \\
\hline Odontesthes bonarensis & 163 & Carriquiriborde, 2011 \\
\hline Poecilia reticulata & $12,01->400$ & Sadeghi et Hedayat, 2014 \\
\hline Cyprinus carpio & $3,26-620$ & Bawa et al., 2017 \\
\hline Lepomis obtusidens & $>100$ & Glusczak et al., 2006 \\
\hline Aphanius dispar & 115,25 & Ibrahim et Khaled, 2017 \\
\hline Piaractus brachyponus & 97,47 & Ramirez - Duarte et al., 2008 \\
\hline Clarias gariepinus & $0,05-43,65$ & Akinsorotan, 2013 \\
\hline Oreochromis niloticus & $1,05-16,8$ & Jiraungkoorskul et al., 2002 ; Ayoola, 2008 \\
\hline Heteroclarias & 6,838 & Micah et al., 2017 \\
\hline Palloceros caudimaculatus & 975 & Shiogiri et al., 2010 \\
\hline Catla catla & 5,191 & Veeraiah, 2015 \\
\hline Tilapia zillii & 211,80 & Nwani et al., 2013 \\
\hline
\end{tabular}


Tableau 5 : CL50 de quelques invertébrés et amphibiens aquatiques.

\begin{tabular}{|c|c|c|c|}
\hline Espèces & Paramètres d'effet & $\begin{array}{l}\text { Concentrations } \\
(\mathrm{mg} \mathrm{m.a} / \mathrm{L})\end{array}$ & Sources \\
\hline \multicolumn{4}{|l|}{ Invertébrés } \\
\hline Ceriodaphnia dubia & CL50-48 h & 147 & RCQE, 2012 \\
\hline Chironomus plumosus & CL50-48 h & $13-55$ & $\begin{array}{c}\text { US EPA, } 2007 b \text { et } \\
2007 c\end{array}$ \\
\hline Daphnia magna & CL50-48 h & $3-1000$ & $\begin{array}{l}\text { US EPA, 2007a, } \\
2007 b \text { et } 2007 c\end{array}$ \\
\hline Daphnia pulex & CL50 - $48 \mathrm{~h}$ & $7,9-242$ & US EPA, 2007b \\
\hline $\begin{array}{l}\text { Grammarus } \\
\text { pseudolimnaeus }\end{array}$ & CL50 - $48 \mathrm{~h}$ & $42-62$ & US EPA, 2007b \\
\hline Hyatella azteca & CL50 - $96 \mathrm{~h}$ & 144,603 & RCQE, 2012 \\
\hline Mysidopsis bahia & CL50 - $96 \mathrm{~h}$ & 40 & US EPA, 2007a \\
\hline \multicolumn{4}{|l|}{ Amphibiens } \\
\hline Crinia insignifera & CL50 - 96 h & 78 & RCQE, 2012 \\
\hline Litoria moorei & CL50 - $96 \mathrm{~h}$ & $11,6-110,8$ & US EPA, 2007a \\
\hline Rana clamitans & CL50 - $96 \mathrm{~h}$ & 138,9 & Howe et al., 2004 \\
\hline
\end{tabular}

\section{Conclusion}

Il ressort de tout ce qui précède que le glyphosate, le phytocide le plus répandu et le plus utilisé dans le monde et plus singulièrement en Afrique et au Bénin pour contrôler les plantes ravageuses des cultures, est peu toxique sur les animaux aquatiques lorsqu'il est utilisé seul. Sa toxicité dépend, entre autres, de la nature du tensioactif de la formulation, de sa concentration, de l'espèce, son sexe, son stade de développement, des caractéristiques physico-chimiques du milieu de vie de cette espèce. Il importe donc d'entreprendre, dans nos conditions d'utilisation de cet herbicide, des études plus précises sur son impact sur la faune aquatique et sur les écosystèmes aquatiques, réceptacles finaux des polluants.

\section{CONFLIT D'INTERETS}

Aucun conflit d'intérêts concernant ce travail.

\section{CONTRIBUTIONS DES AUTEURS}

PA a participé à la conception de la thématique, à la recherche documentaire et à la rédaction de l'article. IIT a participé à la conception de la thématique, à la recherche documentaire et à la correction de l'article.

\section{REMERCIEMENTS}

Les auteurs remercient sincèrement HOUNDJI Alexis et DEGILA Bodelaire respectivement Doctorant à l'Université de Namur et Mastorant à l'Université de Liège en Belgique, qui ont contribué d'une manière ou d'une autre à la recherche documentaire.

\section{REFERENCES}

ACTA (Association de Coordination Technique Agricole). 2010. Index Phytosanitaire 2011 (47 $7^{\mathrm{ème}}$ édn). ACTA : Paris.

Adechian SA, Baco MN, Akponikpe I, Toko II, Egah J, Affoukou K. 2015. Les pratiques paysannes de gestion des pesticides sur le maïs et le coton dans le bassin cotonnier du Bénin. Revue Electronique en Sciences de l'Environnement, 15(2). DOI: 10.4000/vertigo. 16534

Agbohessi TP, Imorou Toko I, Yabi JA, Dassoundo-Assogba JFC, Kestemont P. 2011. Caractérisation des pesticides chimiques utilisés en production 
cotonnière et impact sur les indicateurs économiques dans la Commune de Banikoara au nord du Bénin. Int. J. Biol. Chem. Sci., 5(5): 1828-1841. DOI: 10.4314/ijbcs.v5i5.6

Agbohessi TP, Imorou Toko I, Attakpa EY, Kestemont P. 2012. Synthèse des caractéristiques physico-chimiques et effets écotoxicologiques du Tihan 175 OTEQ utilisé dans la protection phytosanitaire du cotonnier au Bénin. Int. J. Biol. Chem. Sci., 6(5): 2280-2292. DOI: 10.4314/ijbcs.v6i5.34

Agbohessi TP, Imorou Toko I, Atchou V, Tonato R, Mandiki SNM, Kestemont P. 2015. Pesticides used in cotton production affect reproductive development, endocrine regulation, liver status and offspring fitness in African catfish Clarias gariepinus (Burchell, 1822). Comp. Biochem. Physiol., 167: 157-172. DOI: 10.1016/j.cbpc.2014.10.002

Aïkpo FH, Chabi CB, Ahy V, Koumolou L, Houssou CS, Edorh PA. 2015. Evaluation de la contamination des eaux du fleuve Couffo dans la zone cotonnière de Djidja (Bénin) par les pesticides. Int. J. Biol. Chem. Sci., 9(3): 1725-1732. DOI:10.4314/ijbcs.v9I3.50

Akinsorotan AMS, Zelibe AA, Olele NF. 2013. Histopathological effects of acutely toxic levels of dizensate (glyphosate herbicide) on gill and liver of Clarias gariepinus adult. Inter. J. Engi. Res., 4(3): 22295518.

Al-Kawaz JM. 2019. Effect of acute toxicity of glyphosate in gold fish Carassius auratus. Ann. Trop. Med. Publ. Health, 22: SP173. DOI: http://doi.org/10.36295/ASRO.2019.220 517

Ani LC, Nwamba HO, Ejilibe CO, Nwani CD. 2017. Acute toxicity of glyphosate-based herbicide Glycot on juvenile african cat fish Clarias gariepinus (Burchell 1822).
J. Fish Livest Prod., 5(3): 252. DOI: 10.4172/2332-2608.1000252

Arraj T. 2015. Conception d'un système d'élimination du glyphosate des eaux de drainage agricole. Thèse de Doctorat en Géoscience, Université de Lorraine, Lorraine, $119 \mathrm{p}$.

Ayoola SO. 2008. Toxicity of glyphosate herbicide on Nile tilapia (Oreochromis niloticus) juvenile. Afric. J. Agric. Res., 3(12): 825-834.

Bawa VK, Kondal SS, Hundal HK. 2017. Biochemical and histological effects of glyphosate on the liver of Cyprinus carpio. Amer J. Life Sci., 5: 71-80. DOI: 10.11648/j.ajls.s.2017050301.21

Benbrook CM. 2016. Trends in glyphosate herbicide use in the United States and globally. Environ. Sci. Eur., 28: 1-15. DOI: 10.1186/s12302-016-0070-0

Borjesson E, Torstensson L. 2000. New methods for determination of glyphosate and (aminomethyl) phosphonic acid in water and soil. J. Chromatogr A., 886 (12): 207-216. DOI: $10.1016 / \mathrm{s} 0021-$ 9673(00)00514-8

Bowmer KH, Boulton MD, Short DL, Higgins ML. 1986. Glyphosate: Sediment interactions and phytotoxicity in turbid water. Pest. Sci., 17: 79-88. DOI: https://doi.org/10.1002/ps.2780170203

Bridi D, Altenhofen S, Gonzalez JB, Reolon GK, Bonan CD. 2017. Glyphosate and Roundup ${ }^{\circledR}$ alter morphology and behavior in zebrafish. Toxicol., 392: 3239. DOI: $10.1016 /$ j.tox.2017.10.007

Carriquiriborde P. 2011. Toxicidad de glifosato en peces autóctonos: Estudios de laboratorio y campo. In Aspectos Ambientales Del Uso Del Glifosato, Camino M, Aparicio V (Eds). Ediciones INTA: Argentina; 57-68.

Çavas T, Konen S. 2007. Detection of cytogenetic and DNA damage in peripheral erythrocytes of goldfish (Carassius auratus) exposed to a glyphosate formulation using the 
micronucleus test and the cornet assay. Mutagenesis, 22(4): 263- 268. DOI: 10.1093/mutage/gem012

CCME (Conseil Canadien des Ministres de l'Environnement). 2012. Scientific criteria document for the development of the canadian water quality guidelines for the protection of aquatic life Glyphosate. CCME, Winnipeg-Canada.

Costa M, Monteiro DA, Oliveira-Neto AL, Rantin PT, Kalinin AL. 2008. Oxidative stress biomarkers and heart function in bullfrog tadpoles exposed to Roundup original ${ }^{\circledR}$. Ecotoxicol., 17(3): 153-163. DOI: $10.1007 / \mathrm{s} 10646-007-0178-5$

Do Carmo Langiano V, Martinez EBR. 2008. Toxicity and effects of a glyphosate based herbicide on the Neotropical fish Prochilodus lineatus. Comp. Biochem. Physiol., 147(2): 222-231. DOI: 10.1016/j.cbpc.2007.09.009

Dornelles MF, Oliveira GT. 2015. Toxicity of atrazine, glyphosate, and quinclorac in bullfrog tadpoles exposed to concentrations below legal limits. Environ. Sci. Pollut. Res., 23(2): 16101620. DOI : 10.1007/s11356-015-5388-4

Druart C, Millet M, Scheifler R, Delhomme O, de Vaufleury A. 2011. Glyphosate and glufosinate-based herbicides: Fate in soil, transfer to, and effects on land snails. $J$. Soils Sediments, 11(8): 1373-1384. DOI: 10.1007/s11368-011-0409-5

El-Shebly AA, El-kady MAH. 2008. Effects of glyphosate herbicide on serum growth hormone (GH) levels and muscle protein content in Nile tilapia (Oreochromis niloticus L.). Res. J. Fish. Hydrobiol., 3(2): 84-88.

Erhunmwunse NO, Ewere EE, Ainerua MO, Alohan FI. 2014. Biomarker of exposure: alterations in GIT of post juvenile Africa catfish (Clarias gariepinus) exposed to sub-lethal concentrations of glyphosate herbicide (IPA 360g/L). Int. J. Innov. Sci. Res., 1(4): 1-5.
European Commission. 2002. Review report for the active substance glyphosate. 6511/VI/99-final, 1-56. European Commission, Commission working document, France.

Faure G, Fok M, Rollin D, Diakité CH, Koné M, Beauval V, De Noray S, Dembélé D. 2000. Etude de faisabilité d'un programme d'amélioration des systèmes d'exploitation en zone cotonnière : Rapport final mai 2000. CIRAD-TERA, Montpellier, $100 \mathrm{p}$.

Folmar LC, Sanders HO, Julin AM. 1979. Toxicity of the herbicide glyphosate and several of its formulations to fish and aquatic invertebrates. Arch. Environ. Contam. Toxicol., 8: 269-278. DOI: https://doi.org/10.1007/BF01056243

Gbaguidi MAN, Soclo HH, Issa YM, Fayomi $\mathrm{B}$, Dognon R, Agagbé A, Bonou C, Youssao A, Dovonou LF. 2011. Evaluation quantitative des résidus de pyréthrinoïdes, d'aminophosphate et de triazines en zones de production de coton au Bénin par la méthode ELISA en phase liquide: cas des eaux de la rivière Agbado. Int. J. Biol. Chem. Sci., 5(4): 1476-1490. DOI: 10.4314/ijbcs.v5i4.14

Glusczak L, Miron DS, Pedron FA, Vieira VLP. 2006. Effect of glyphosate herbicide on acetylcholinesterase activity and metabolic and haematological parameters in Piava (Leporinus obtusidens.). Ecotoxicol. Environ. Saf., 65: 237-241. DOI: 10.1016/j.ecoenv.2005.07.017

Glusczak L, Miron DS, Moraes BS, Simmoes RR, Schetinger MRC, morschV, Loro VL. 2007. Acute effects of glyphosate herbicide on metabolic an enzymatic parameters of silver catfish (Rhamdia quelen). Comp. Biochem. Physiol., 146: 519-524.

DOI: 10.1016/j.cbpc.2007.06.004

Goldsborough LG, Brown DJ. 1993. Dissipation of glyphosate and aminomethylphosphonic acid in water 
and sediments of boreal forest pond. Environ. Toxicol. Chem., 12: 1139-1147. DOI: $10.1002 /$ etc.5620120702

Govindarajulu PP. 2008. Literature review of impacts of glyphosate herbicide on amphibians: What risks can the silvicultural use of this herbicide pose for amphibians in B.C. Ministry of Environment Wildlife Report, Victoria.

Guo ZX, Cai Q, Yang Z. 2005. Determination of glyphosate and phosphate in water by ion chromatography, inductively coupled plasma mass spectrometry detection. $J$. Chromatogr. A., 1100(2): 160-167. DOI: 10.1016/j.chroma.2005.09.034

Howe CM, Berrill M, Pauli BD, Helbing CC, Werry K, Veldhoen N. 2004. Toxicity of glyphosate-based pesticides to four North American frog species. Environ. Toxicol. Chem., 23(8): 1928-1938. DOI: 10.1897/03-71

Hued AC, Oberhofer S, Bistoni MD. 2012. Exposure to a commercial glyphosate formulation (Roundup®) alters normal gill and liver histology and affects male sexual activity of Jenynsia multidentata (Anablepidae, Cyprinodontiformes). Arch. Environ. Contam. Toxicol., 62(1): 107-117. DOI: $10.1007 / \mathrm{s} 00244-011-$ 9686-7

Ibrahim AM, Khaled AAZ. 2017. Evaluation of Glyphosate toxicity on Arabian killifish, Aphanius dispar Collected from Southwestern Saudi Arabia. Global J. Sci. Frontier Res., 17(3): 44-50.

INERIS (Institut National de l'Environnement Industriel et des Risques). 2011. Normes de qualité environnementale, Glyphosate$\mathrm{n}^{\circ}$ cas : 1071-83-6. INERIS, France, $17 \mathrm{p}$. INERIS. 2013. Valeur guide environnementale, Glyphosate $-\mathrm{n}^{\circ}$ cas : 1071-83-6. INERIS, France, 22 p.

INRS (Institut National de la Recherche et de Sécurité). 2019. Base de données, fiches toxicologiques 273. INRS, France, 9 p. http://www.inrs.fr/accueil/produits/bdd/d oc/fichetox.html?refINRS=FT\%20273
Jerald FF, Saradhamani N, ManishKumar B, Prabhu CMF. 2016. Toxic effects of herbicide glyphosate Hijack (41\%) on biochemical parameters of freshwater fish, Catla catla (HAM). World J. Pharm. Pharm. Sci., 5(7): 1928-1937. DOI: 10.20959/wjpps20167-7271

Jiraungkoorskul W, Upathama ES, Kruatrachuea M, Sahaphongc S, Vichasri- Gramsa S, Pokethitiyooka P. 2002. Histopathological effects of Roundup, a glyphosate herbicide, on Nile tilapia (Oreochromis niloticus). Sci. Asia, 28: 121-127. DOI: 10.2306/scienceasia15131874.2002.28.121

Jiraungkoorskul W, Upatham ES, Kruatrachue M, Sahaphong S, Vichasri-Grams S, Pokethitiyook P. 2003. Biochemical and histopathological effects of glyphosate herbicide on Nile tilapia (Oreochromis niloticus). Environ. Toxicol., 18(4): 260267. DOI: $10.1002 /$ tox. 10123

Katrijn MAH, Piet S, Peter AV. 2007. Monitoring and modelling pesticide fate in surface water at the catchment scale. Ecol. Modell., 209: 53-64. DOI:10.1016/j.ecolmodel.2007.07.030

Kennedy C. 2017. Glyphosate fate and toxicity to fish with special relevance to salmon and steelhead populations in the Skeena River watershed. Report, $55 \mathrm{p}$.

Larvor-Cario H, de Laborie LP, Hureau D, Muzellec ML, Devauchelle N, Durand G, Dorange G. 2000. Influence d'herbicides détectés en rade de Brest sur le développement embryonnaire et larvaire de la coquille Saint-Jacques Pecten maximus. Rapport Ifremer / Ecloserie du Tinduff / Institut de synergie des Sciences et de la Santé, Grande Bretagne.

Le Mer C. 2009. Effets des expositions aux herbicides atrazine et glyphosate sur les larves d'épinoche à trois épines Gasterosteus aculeatus. Mémoire de Maitrise en Océanographie, Université de Québec, Québec, 120 p. 
MAEP (Ministère de l'Agriculture de l'Elevage et de la Pêche). 2015. Statistiques agricoles. Cotonou : DPP/MAEP. MAEP, Benin.

Marc J, Mulner-Lorillon O, Boulben S, Hure D, Durand G, Bellé R. 2002. Pesticide Roundup provokes cell Division dysfunction at the level of CDK1/cyclin B activation. Chem. Res. Toxicol., 15(3): 326-331. DOI: 10.1021/tx015543

Messaad IA, Al Zailaie KA. 2017. Evaluation of glyphosate toxicity on Arabian killifish, Aphanius dispar collected from Southwestern Saudi Arabia. Global J. Sci. Frontier, 17(3):43-50.

Micah AD, Adakole JA, Yusuf A, Mohammed NA. 2017. Acute effects of glyphosate on the behavioural and hematological characteristics of heteroclarias (hybrid) fingerlings. J. Aquacult. Eng. Fish. Res., 3(1): 13-18. DOI: 10.3153/JAEFR17003

Mitchell DG, Chapman PM, Long TJ. 1987. Acute toxicity of Roundup and Rodeo herbicides to rainbow trout, Chinook, and coho salmon. Bull. Environ. Contam. Toxicol., 39(6): 1028-1035. DOI: 10.1007/BF01689594

Naili F. 2014. Evaluation de la rémanence de l'herbicide Glyphosate dans les cultures maraîchères de la wilaya de Jijel. Mémoire de Magister en Biologie appliquée, Université Constantine 1, Algérie, $114 \mathrm{p}$.

Nwani CD, Ibiam UA, Ibiam OU, Nworie O, Onyishi G, Atama C. 2013. Investigation on acute toxicity and behavioral changes in Tilapia zillii due to glyphosate-based herbicide, force up. J. Ani. Plant. Sci., 23(3): 888 - 892.

Obiora FO. 2008. Possible toxic effects of glyphosate based herbicide, Roundup on fish exposed to laboratory doses. Thèsede Doctorat, Université du Nigéria, Nigéria, $102 \mathrm{p}$.

Okwara N, Ibekwe C, Madubuike KG. 2016. Clinical and hematological effects of glyphosate herbicide on Clarias gariepinus juveniles. Vet Adv., 6(4): 1237-1241.

DOI: 10.5455/jva.20160323060011

Paunescu A, Ponepal CM. 2011. Effect of Roundup ${ }^{\circledR}$ Herbicide on physiological indices in marsh frog Pelophylax ridibundus. Agronomy, 54 : 269-274.

Picqué A. 2016. Evaluation des impacts du Glyphosate sur la santé humaine. Thèse de Doctorat d'Etat en Pharmacie, Université de Picardie, Picardie, 66 p.

Rainaud PL. 2013. Evaluation des risques à long terme des herbicides à base du glyphosate sur la santé humaine. Thèse de Doctorat d'Etat, Université de Limoges, Limoges, $183 \mathrm{p}$.

Ramesh M, Srinivasan R, Saravanan M. 2009. Effect of atrazine (Herbicide) on blood parameters of common carp Cyprinus carpio (Actinopterygii: Cypriniformes). Afric. J. Environ. Sci. Technol., 3(12): 453-458.

Ramírez-Duarte WF, Rondón-Barragán LS, Eslava-Mocha PR. 2008. Acute toxicity and histopathological alterations of Roundup ${ }^{\circledR}$ herbicide on "cachama blanca" (Piaractus brachypomus). Pesq. Vet. Bras., 28(11): 547-554. DOI: 10.1590/S0100-736X2008001100002

RCQE (Recommandations canadiennes pour la qualité des eaux : protection de la vie aquatique). 2012. Glyphosate. RCQE, Arabie saoudite, $11 \mathrm{p}$.

Royer A, Beguin S, Tabet JC, Hulot S, Reding MA, Communal PY. 2000. Determination of glyphosate and aminomethylphosphonic acid residues in water by gas chromatography with tandem mass spectrometry after exchange ion resin purification and derivatization: application on vegetable matrixes. Anal. Chem., 72(16): 3826-3832. DOI: 10.1021/ac000041d

Sadeghi A, Hedayati A. 2014. Investigation of LC50, NOEC and LOEC of Glyphosate, Deltamethrin and Pretilachlor in Guppies 
(Poecilia Reticulata). Iranian J. Toxicol., 8(26) : 1124-1129.

Schuette J. 1998. Environmental fate of glyphosate. Environmental Monitoring \& Pest Management, Department of Pesticide Regulation Sacramento, 1-13.

Shiogiri NS, Cubo P, Schiavetti C, Pitelli RA. 2010. Ecotoxicity of glyphosate and aterbane (B) by surfactant on guaru (Phalloceros caudimaculatus). J. Anim. Biol. Sci., 32: 285-289. DOI: http://dx.doi.org/10.4025/actascibiolsci.v 32i3.6795

Smith GR. 2001. Effects of acute exposure to a commercial formulation of glyphosate on the tadpoles of two species of anurans. Bull. Environ. Contam. Toxicol., 67(4): 483-488. DOI: 10.1007/s001280149

Solomon KR, Thompson DG. 2003. Ecologicol risk assessment for aquatiquc organisms from over-water uses of glyphosate. J. Toxicol. Env. Heal. B Crit. Rev., 6(3): 289-324. DOI: $10.1080 / 10937400306468$

Stalikas CD, Konidari CN. 2001. Analytical methods to determine phosphonic and amino acid group-containing pesticides. J. Chromatogr. A., 907(1-2): 1-19. DOI: 10.1016/s0021-9673(00)01009-8

Struger J, Thompson D, Staznik B, Martin P, McDaniel T, Martin C. 2008. Occurrence of glyphosate in surface waters of southern Ontario. Bull. Environ. Contam. Toxicol., 80: 378-384. DOI: 10.1007/s00128-008-9373-1

Summit Environmental Consultants Ltd. 2007. Toxicity of glyphosate and thiram to aquatic invertebrates. Final report submitted to national guidelines and standards office, Canada, $24 \mathrm{p}$.

Tate TM, Spurlock IO, Christian FA. 1997. Effect of glyphosate on the development of Pseudosuccinea columella snails. Arch. Environ. Contam. Toxicol., 33: 286-289. DOI: $10.1007 / \mathrm{s} 002449900255$
Tooby TE. 1985. Fate and biological consequences of glyphosate in the aquatic environment. In The Herbicide Glyphosate, Grossbard E, Atkinson D (eds). Butter worths: London; 206-217.

Traas TP, Smit CE. 2003. Environmental risk limits for aminomethylphosphonic acid (AMPA). RIVM report 601501018/2003, 23 p.

Tsui MTK, Chu LM. 2003. Aquatic toxicity of glyphosate-based formulations: comparison between different organisms and the effects of environmental factors. Chemosphere, 52: 1189-1197. DOI: 10.1016/S0045-6535(03)00306-0

USEPA (United States Environmental Protection Agency). 2005. Pesticide Ecotoxicity Database, Environmental Fate and Effects Division of the Office of Pesticide Programs. USEPA, United States.

USEPA. 2007a. USEPA. US EPA, Ecotoxicology Database. USEPA, United States.

USEPA. 2007b. USEPA. US EPA, Pesticide Fate Database. USEPA, United States.

USEPA. 2007c. USEPA. US EPA, Restricted Database. USEPA, United States.

USEPA. 2008. EPA's 2008 Report on the Environment. National Center for Environmental Assessment, Washington, DC. USEPA, United States. http://www.epa.gov/roe

Veeraiah K, Padmaja B, Sai RV, Naga PM, Vivek Ch. 2015. Impact of glyphosate on biochemical constituents of the freshwater fish, Catla catla. Int. J. Bioassays, 4(07): 4139-4144. DOI: 10.21746/IJBIO.2015.07.0021

Wang YS, Jaw CG, Chen YL. 1994. Accumulation of 2,4-D and glyphosate in fish and water hyacinth. Water Air and Soil Poll., 74: 397-403. DOI: 10.1007/BF00479802 